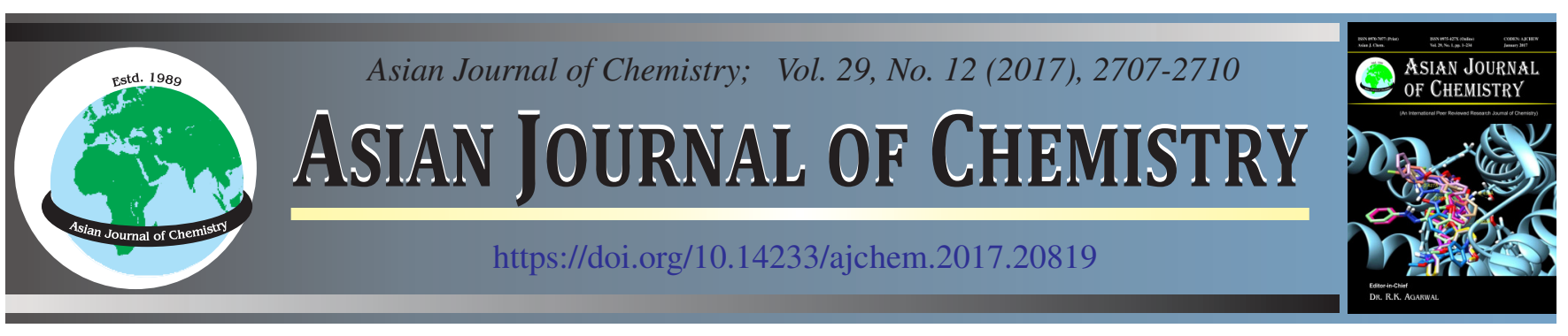

\title{
Synthesis and Spectroscopic Investigations of Schiff Bases Transition Metal Complexes of Copper(II), Cobalt(II) and Nickel(II)
}

\author{
RAJESHWAR RAI ${ }^{1}$, RitIKA KUMARI ${ }^{2}$ and B.K. RAI ${ }^{3, *}$
}

${ }^{1}$ Department of Chemistry, Darbhanga College of Engineering, Darbhanga-846 005, India

${ }^{2}$ Department of Chemistry, Ram Shrestha Singh College, Chochahan, Muzaffarpur-844 111, India

${ }^{3}$ Department of Chemistry, Lalit Narayan Tirhut College, Muzaffarpur-842 001, India

*Corresponding author: E-mail: binodkr_rai@yahoo.co.in

Received: 26 May 2017;

Accepted: 31 July 2017;

Published online: 30 October 2017;

AJC-18620

This work presents an experimental and theoretical study on complexes of copper(II), cobalt(II) and nickel(II) with Schiff base ligand 3-propyl-(N-o-toluidine)quinazoline semicarbazone (PTQS). The spectroscopic properties of the complexes have been investigated by IR and electronic spectra. The molecular geometry of the complexes have been proposed on the basis of elemental analyses, molar mass and magnetic susceptibility. It is suggested that the ligand PTQS acts in a bidentate manner and coordination proposes with azomethine $\mathrm{N}$ and carbonyl oxygen atom of semicarbazone moiety. The remaining coordination sites are occupied by negative ions such as $\mathrm{Cl}^{-}, \mathrm{Br}^{-}, \mathrm{I}^{-}, \mathrm{NO}_{3}^{-}$ and $\mathrm{ClO}_{4}^{-}$. The geometry of complexes was assigned to be octahedral.

Keywords: 3-Propyl-(N-o-toludine)quinazoline, Semicarbazone, Schiff base, Transition metal(II) complexes.

ᄂ _ - - - - - - - - - - - - - - - - - - - - - - - - -

\section{INTRODUCTION}

The increasing growth of coordination chemistry of Schiff base during last two decades have contributed to great success in various fields and drawn immense attention due to their versatile applications [1-5]. These complexes show oxidase biometric catalytic activity, anticancer and antimicrobial activities [6-11]. The study of Schiff bases and their complexes has become a focal point of interest for chemists in recent years, not only for their several structural features, but due to their potential application in various fields [12-15].

Transition metal complexes with Schiff base ligands are reported by several authors [16-20]. In the light of bioactivity of Schiff bases and their metal coordination compounds and in continuation of our previous recent work [21-25] in this field, a series of complexes have been synthesized and characterized by using transition metal complexes of $\mathrm{Co}$ (II), $\mathrm{Ni}$ (II) and $\mathrm{Cu}$ (II) with Schiff base ligand 3-propyl-(N-o-toluidine)quinazoline semicarbazone (PTQS).

\section{EXPERIMENTAL}

The synthetic reactions and workup were performed in open air at room temperature. All other chemicals and solvent used were analytical reagent grade and used without further purification. Elemental analyses (carbon, nitrogen, hydrogen and metal ions) were performed on a Perkin-Elmer $2400 \mathrm{CHNO}$ elemental analyzer.

The ligand as well as metal complexes were analyzed by using standard method [26]. The IR spectra of ligand and its metal complexes were recorded on Beckman IR20 Infrared Spectrophotometer using $\mathrm{KBr}$ pellets. The electronic spectra of the complexes were recorded on a Cary 2390 Spectrophotometer. Magnetic moment was measured by Gouy balance using $\mathrm{Hg}\left[\mathrm{Co}(\mathrm{NCS})_{4}\right]$ as a calibrant. The molar conductance were performed on Toshniwal conductivity bridge using DMF as solvent.

Preparation of ligand: Ethanolic solution of 3-methyl (N-o-toluidine)quinazoline $(0.01 \mathrm{~mol})$ was allowed to react with semicarbazide hydrochloride $(0.01 \mathrm{~mol})$ dissolved in sodium acetate. The resulting reaction mixture was heated on water bath for 3-4 h with frequent stirring. After cooling, the preci-pitate was collected, washed with ether and treated with dilute sodium carbonate solution and filtered. The solid was washed thoroughly with ethanol to yeild 3-methyl (N-o-toludine)quinazoline semicarbazone (PTQS) as colourless compound (m.p. $167 \pm 10^{\circ} \mathrm{C}$. Yield 70-75\%).

Preparation of the metal complexes: The complexes of $\mathrm{Co}(\mathrm{II}), \mathrm{Ni}(\mathrm{II})$ and $\mathrm{Cu}(\mathrm{II})$ have been prepared by treating an ethanolic solution of respective metal halide/nitrate/perchlorate with ethanolic solution of the ligand PTQS in molar ratio 1:2. 
The resulting reaction mixture was refluxed on water bath per 2-3 $\mathrm{h}$. The procedure carried out in each case of similar nature with a slight variation of timing of reflux. The solid coloured complexes obtained in each case were filtered and washed with ethanol several times to remove any excess of the ligand. Finally, complex was washed with anhydrous diethyl ether (Yield: 75$80 \%)$.

\section{RESULTS AND DISCUSSION}

On the basis of study of molar mass, infrared spectral data, it may be proposed that the ligand ligand 3-methyl (N-otoluidine)quinazoline semicarbazone (PTQS) acts in a neutral bidentate manner. The remaining coordination centres of metal ions are satisfied by negative ions such as $\mathrm{Cl}^{-}, \mathrm{Br}^{-}, \mathrm{I}^{-}, \mathrm{NO}_{3}^{-}$ and $\mathrm{ClO}_{4}^{-}$(Table-1).

The IR spectra of ligand 3-methyl ( $\mathrm{N}-\mathrm{o}$-toluidine)quinazoline semicarbazone (PTQS) exhibit a broad band of medium intensity at $1480 \mathrm{~cm}^{-1}$ can be assigned to $\mathrm{C}=\mathrm{N}$ [27-29]. In the spectra of the complex, this band shows red shift with slightly reduced intensity. The shift of the band and change in intensity proposes coordination of azomethine-N with metal ion. The infrared spectra of the ligand PTQS shows a strong and broad band at $1760 \mathrm{~cm}^{-1}$ assignable to $\mathrm{C}=\mathrm{O}$ [27-30]. This band undergoes to shift after complex formation proposes coordination to the metal ion through carbonyl oxygen (Table-2).
The linkage with ligand to metal ion through oxygen atom of either nitrate or perchlorate ion, $\mathrm{N}$-atom of azomethine group and carbonyl oxygen of semicarbazone moiety is supported by the appearance of bands due to $\mathrm{v}(\mathrm{M}-\mathrm{O})$ at $530-505 \mathrm{~cm}^{-1}, \mathrm{v}(\mathrm{M}-\mathrm{N})$ at $435-410 \mathrm{~cm}^{-1}$, respectively $[27,28,31]$. The evidence of metalhalogen is supported by the low molar conductance values of the complexes in the range 4.9-6.7 $\Omega \mathrm{cm}^{2} \mathrm{~mol}^{-1}$ [32] and appearance of a band in the region $325-265 \mathrm{~cm}^{-1}$ which assigned to $v(\mathrm{M}-\mathrm{X})[27,28,31]$.

Nitrate complexes show characteristic medium intensity bands of 1320 and $1160 \mathrm{~cm}^{-1}$ with a separation of $160 \mathrm{~cm}^{-1}$ due to monodentate linkage of nitrate group [33]. Combination band at 1580 and $1560 \mathrm{~cm}^{-1}$ with a separation of $20 \mathrm{~cm}^{-1}$ confirming the monodentate behaviour of the nitrate group. The monodentate behaviour of perchlorate complexes were supported by the presence of four key IR bands at 1125, 1045, 655 and $625 \mathrm{~cm}^{-1}$ suggesting monodentate behaviour of perchlorate group [34].

Electronic spectral and magnetic susceptibility: The cobalt(II) complexes exhibit three bands in the region 1030010140, 12900-13200 and 20600-22060 $\mathrm{cm}^{-1}$ assigned to ${ }^{4} \mathrm{~T}_{2 \mathrm{~g}}$ $\leftarrow{ }^{4} \mathrm{~T}_{1 \mathrm{~g}}(\mathrm{~F}),{ }^{4} \mathrm{~A}_{2 \mathrm{~g}}(\mathrm{~F}) \leftarrow{ }^{4} \mathrm{~T}_{1 \mathrm{~g}}(\mathrm{~F})$ and ${ }^{4} \mathrm{~T}_{1 \mathrm{~g}}(\mathrm{P}) \leftarrow{ }^{4} \mathrm{~T}_{1 \mathrm{~g}}(\mathrm{~F})$, respectively, indicating octahedral geometry of the $\mathrm{Co}(\mathrm{II})$ complexes [35]. The proposed geometry of $\mathrm{Co}$ (II) complex is further supported by the high magnetic susceptibility in the range 4.70-5.01 B.M. The Ni(II) complexes exhibits three bands at 11680-11210, 16400-16260 and 24100-23060 $\mathrm{cm}^{-1}$ attributing ${ }^{3} \mathrm{~T}_{2 \mathrm{~g}}(\mathrm{~F}) \leftarrow$

TABLE-1

ANALYTICAL, COLOUR, MAGNETIC SUSCEPTIBILITY, MOLAR CONDUCTANCE AND DECOMPOSITION TEMPERATURE OF LIGAND PTQS AND ITS METAL COMPLEXES

\begin{tabular}{|c|c|c|c|c|c|c|c|c|}
\hline \multirow{2}{*}{$\begin{array}{l}\text { Compounds } \\
\text { (Colour) }\end{array}$} & \multicolumn{4}{|c|}{ Elemental analysis (\%): Found (calcd.) } & \multirow{2}{*}{$\begin{array}{c}\mu_{\text {eff }} \\
\text { (B.M.) }\end{array}$} & \multirow{2}{*}{$\begin{array}{c}\lambda_{\max } \text { electronic } \\
\left(\mathrm{cm}^{-1}\right)\end{array}$} & \multirow{2}{*}{$\begin{array}{l}\Omega_{\mathrm{m}}\left(\mathrm{ohm}^{-1}\right. \\
\left.\mathrm{cm}^{2} \mathrm{~mol}^{-1}\right)\end{array}$} & \multirow{2}{*}{$\begin{array}{l}\text { Decomposition } \\
\text { temp. }\left({ }^{\circ} \mathrm{C}\right)\end{array}$} \\
\hline & $\mathrm{M}$ & $\mathrm{C}$ & $\mathrm{N}$ & $\mathrm{H}$ & & & & \\
\hline $\begin{array}{l}\text { PTQS } \\
\text { (Colourless) }\end{array}$ & - & $\begin{array}{c}77.53 \\
(77.69)\end{array}$ & $\begin{array}{c}12.91 \\
(12.96)\end{array}$ & $\begin{array}{c}8.27 \\
(8.33)\end{array}$ & - & - & - & - \\
\hline $\begin{array}{l}{\left[\mathrm{Co}(\mathrm{PTQS})_{2} \mathrm{Cl}_{2}\right]} \\
\text { (Yellowish red) }\end{array}$ & $\begin{array}{l}7.30 \\
(7.36)\end{array}$ & $\begin{array}{c}31.90 \\
(32.00)\end{array}$ & $\begin{array}{c}17.38 \\
(17.50)\end{array}$ & $\begin{array}{l}3.95 \\
(4.00)\end{array}$ & 4.86 & $\begin{array}{c}10230,13200, \\
22060\end{array}$ & 5.3 & 243 \\
\hline $\begin{array}{l}{\left[\mathrm{Co}(\mathrm{PTQS})_{2} \mathrm{Br}_{2}\right]} \\
\text { (Yellowish red) }\end{array}$ & $\begin{array}{c}6.57 \\
(6.63)\end{array}$ & $\begin{array}{l}28.65 \\
(28.79)\end{array}$ & $\begin{array}{c}15.63 \\
(15.75)\end{array}$ & $\begin{array}{c}3.54 \\
(3.60)\end{array}$ & 4.89 & $\begin{array}{c}10210,12900, \\
20800\end{array}$ & 5.1 & 260 \\
\hline $\begin{array}{l}{\left[\mathrm{Co}(\mathrm{PTQS})_{2} \mathrm{I}_{2}\right]} \\
\text { (Dark brown) }\end{array}$ & $\begin{array}{c}5.93 \\
(5.99)\end{array}$ & $\begin{array}{l}25.89 \\
(26.04)\end{array}$ & $\begin{array}{c}14.18 \\
(14.24)\end{array}$ & $\begin{array}{c}3.19 \\
(3.25)\end{array}$ & 4.79 & $\begin{array}{c}10140,13060, \\
20680\end{array}$ & 4.9 & 250 \\
\hline $\begin{array}{l}{\left[\mathrm{Co}(\mathrm{PTQS})_{2}\left(\mathrm{NO}_{3}\right)_{2}\right]} \\
\text { (Dark brown) }\end{array}$ & $\begin{array}{c}6.84 \\
(6.90)\end{array}$ & $\begin{array}{c}29.13 \\
(30.01)\end{array}$ & $\begin{array}{c}16.28 \\
(16.41)\end{array}$ & $\begin{array}{c}3.71 \\
(3.75)\end{array}$ & 4.83 & $\begin{array}{c}10260,13140, \\
20600\end{array}$ & 5.2 & 245 \\
\hline $\begin{array}{l}{\left[\mathrm{Co}(\mathrm{PTQS})_{2} \mathrm{ClO}_{4}\right]} \\
\text { (Light yellow) }\end{array}$ & $\begin{array}{c}6.29 \\
(6.35)\end{array}$ & $\begin{array}{c}27.43 \\
(27.58)\end{array}$ & $\begin{array}{c}14.87 \\
(15.08)\end{array}$ & $\begin{array}{c}3.40 \\
(3.44)\end{array}$ & 5.01 & $\begin{array}{c}10300,13120 \\
20470\end{array}$ & 5.3 & 273 \\
\hline $\begin{array}{l}{\left[\mathrm{Ni}(\mathrm{PTQS})_{2} \mathrm{Cl}_{2}\right]} \\
\text { (Light yellow) }\end{array}$ & $\begin{array}{c}7.28 \\
(7.34)\end{array}$ & $\begin{array}{c}31.89 \\
(32.01)\end{array}$ & $\begin{array}{c}14.34 \\
(17.50)\end{array}$ & $\begin{array}{c}3.95 \\
(4.00)\end{array}$ & 3.04 & $\begin{array}{c}11260,16300, \\
23700\end{array}$ & 6.4 & 267 \\
\hline $\begin{array}{l}{\left[\mathrm{Ni}(\mathrm{PTQS})_{2} \mathrm{Br}_{2}\right]} \\
\text { (Light yellow) }\end{array}$ & $\begin{array}{c}6.53 \\
(6.60)\end{array}$ & $\begin{array}{l}28.70 \\
(28.81)\end{array}$ & $\begin{array}{c}15.63 \\
(15.75)\end{array}$ & $\begin{array}{l}3.55 \\
(3.61)\end{array}$ & 3.11 & $\begin{array}{c}11310,16260, \\
233080\end{array}$ & 6.7 & 217 \\
\hline $\begin{array}{l}{\left[\mathrm{Ni}(\mathrm{PTQS})_{2} \mathrm{I}_{2}\right]} \\
\text { (Yellowish brown) }\end{array}$ & $\begin{array}{c}5.93 \\
(5.97)\end{array}$ & $\begin{array}{l}25.89 \\
(26.05)\end{array}$ & $\begin{array}{c}14.09 \\
(14.24)\end{array}$ & $\begin{array}{c}3.17 \\
(3.25)\end{array}$ & 3.16 & $\begin{array}{c}11490,16180, \\
24100\end{array}$ & 6.3 & 206 \\
\hline $\begin{array}{l}{\left[\mathrm{Ni}(\mathrm{PTQS})_{2}\left(\mathrm{NO}_{3}\right)_{2}\right]} \\
\text { Brownish yellow) }\end{array}$ & $\begin{array}{c}6.75 \\
(6.88)\end{array}$ & $\begin{array}{c}29.90 \\
(30.02)\end{array}$ & $\begin{array}{c}14.30 \\
(16.41)\end{array}$ & $\begin{array}{c}3.69 \\
(3.75)\end{array}$ & 3.14 & $\begin{array}{c}11680,16400, \\
23060\end{array}$ & 6.1 & 203 \\
\hline $\begin{array}{l}{\left[\mathrm{Ni}(\mathrm{PTQS})_{2} \mathrm{ClO}_{4}\right]} \\
\text { (Light yellow) }\end{array}$ & $\begin{array}{l}6.16 \\
(6.32)\end{array}$ & $\begin{array}{l}27.46 \\
(27.59)\end{array}$ & $\begin{array}{c}14.89 \\
(15.09)\end{array}$ & $\begin{array}{c}3.38 \\
(3.44)\end{array}$ & 3.15 & $\begin{array}{c}11210,16320 \\
23000\end{array}$ & 5.9 & 219 \\
\hline $\begin{array}{l}{\left[\mathrm{Cu}(\mathrm{PTQS})_{2} \mathrm{Cl}_{2}\right]} \\
\text { (Blue) }\end{array}$ & $\begin{array}{c}7.83 \\
(7.89)\end{array}$ & $\begin{array}{c}31.70 \\
(31.81)\end{array}$ & $\begin{array}{c}17.23 \\
(17.40)\end{array}$ & $\begin{array}{c}3.91 \\
(3.97)\end{array}$ & 1.92 & 12480,16200 & 6.2 & 267 \\
\hline $\begin{array}{l}{\left[\mathrm{Cu}(\mathrm{PTQS})_{2} \mathrm{Br}_{2}\right]} \\
\text { (Bluish green) }\end{array}$ & $\begin{array}{c}7.04 \\
(7.11)\end{array}$ & $\begin{array}{c}28.51 \\
(28.65)\end{array}$ & $\begin{array}{c}15.53 \\
(15.67)\end{array}$ & $\begin{array}{c}3.50 \\
(3.58)\end{array}$ & 1.90 & 12600,15800 & 6.5 & 273 \\
\hline $\begin{array}{l}{\left[\mathrm{Cu}(\mathrm{PTQS})_{2}\left(\mathrm{NO}_{3}\right)_{2}\right]} \\
\text { (Deep blue) }\end{array}$ & $\begin{array}{c}7.33 \\
(7.40)\end{array}$ & $\begin{array}{l}29.74 \\
(29.85)\end{array}$ & $\begin{array}{c}16.19 \\
(16.32)\end{array}$ & $\begin{array}{l}3.06 \\
(3.12)\end{array}$ & 1.84 & 12540,15900 & 6.5 & 281 \\
\hline $\begin{array}{l}{\left[\mathrm{Cu}(\mathrm{PTQS})_{2} \mathrm{ClO}_{4}\right]} \\
\text { (Light yellow) }\end{array}$ & $\begin{array}{c}6.76 \\
(6.81)\end{array}$ & $\begin{array}{c}7.39 \\
(7.45)\end{array}$ & $\begin{array}{c}14.92 \\
(15.01)\end{array}$ & $\begin{array}{c}3.36 \\
(3.43)\end{array}$ & 1.81 & 12500,16100 & 5.1 & 287 \\
\hline
\end{tabular}


TABLE-2

SALIENT FEATURES OF IR SPECTRAL DATA $\left(\mathrm{cm}^{-1}\right)$ FOR LIGAND PTQS AND ITS METAL COMPLEXES

\begin{tabular}{|c|c|c|c|c|c|}
\hline Compounds & $v(C=N)$ & $v(\mathrm{C}=\mathrm{O})$ & $v(M-O)$ & $v(\mathrm{M}-\mathrm{N})$ & $v(M-X)$ \\
\hline PTQS & $1480 \mathrm{~s}, \mathrm{~b}$ & $1760 \mathrm{~s}, \mathrm{~b}$ & & & \\
\hline$\left[\mathrm{Co}(\mathrm{PTQS})_{2} \mathrm{Cl}_{2}\right]$ & $1475 \mathrm{~m}, \mathrm{~b}$ & $1740 \mathrm{~m}, \mathrm{~b}$ & $510 \mathrm{~m}$ & $420 \mathrm{~m}$ & $260 \mathrm{~m}$ \\
\hline$\left[\mathrm{Co}(\mathrm{PTQS})_{2} \mathrm{Br}_{2}\right]$ & $1475 \mathrm{~m}, \mathrm{~b}$ & $1740 \mathrm{~m}, \mathrm{~b}$ & $510 \mathrm{~m}$ & $425 \mathrm{~m}$ & $270 \mathrm{~m}$ \\
\hline$\left[\mathrm{Co}(\mathrm{PTQS})_{2} \mathrm{I}_{2}\right]$ & $1475 \mathrm{~m}, \mathrm{~b}$ & $1740 \mathrm{~m}, \mathrm{~b}$ & $505 \mathrm{~m}$ & $425 \mathrm{~m}$ & $275 \mathrm{~m}$ \\
\hline$\left[\mathrm{Co}(\mathrm{PTQS})_{2}\left(\mathrm{NO}_{3}\right)_{2}\right]$ & $1470 \mathrm{~m}, \mathrm{~b}$ & $1735 \mathrm{~m}, \mathrm{~b}$ & $505 \mathrm{~m}$ & $420 \mathrm{~m}$ & \\
\hline$\left.\left[\mathrm{Co}(\mathrm{PTQS})_{2} \mathrm{ClO}_{4}\right)_{2}\right]$ & $1470 \mathrm{~m}, \mathrm{~b}$ & $1740 \mathrm{~m}, \mathrm{~b}$ & $510 \mathrm{~m}$ & $425 \mathrm{~m}$ & \\
\hline$\left[\mathrm{Ni}(\mathrm{PTQS})_{2} \mathrm{Cl}_{2}\right]$ & $1455 \mathrm{~m}, \mathrm{~b}$ & $1735 \mathrm{~m}, \mathrm{~b}$ & $525 \mathrm{~m}$ & $435 \mathrm{~m}$ & $300 \mathrm{~m}$ \\
\hline$\left[\mathrm{Ni}(\mathrm{PTQS})_{2} \mathrm{Br}_{2}\right]$ & $1455 \mathrm{~m}, \mathrm{~b}$ & $1735 \mathrm{~m}, \mathrm{~b}$ & $525 \mathrm{~m}$ & $435 \mathrm{~m}$ & $305 \mathrm{~m}$ \\
\hline$\left[\mathrm{Ni}(\mathrm{PTQS})_{2} \mathrm{I}_{2}\right]$ & $1455 \mathrm{~m}, \mathrm{~b}$ & $1740 \mathrm{~m}, \mathrm{~b}$ & $525 \mathrm{~m}$ & $435 \mathrm{~m}$ & $320 \mathrm{~m}$ \\
\hline$\left[\mathrm{Ni}(\mathrm{PTQS})_{2}\left(\mathrm{NO}_{3}\right)_{2}\right]$ & $1455 \mathrm{~m}, \mathrm{~b}$ & $1735 \mathrm{~m}, \mathrm{~b}$ & $520 \mathrm{~m}$ & $430 \mathrm{~m}$ & \\
\hline$\left[\mathrm{Ni}(\mathrm{PTQS})_{2}\left(\mathrm{ClO}_{4}\right)_{2}\right]$ & $1450 \mathrm{~m}, \mathrm{~b}$ & $1740 \mathrm{~m}, \mathrm{~b}$ & $520 \mathrm{~m}$ & $430 \mathrm{~m}$ & \\
\hline$\left[\mathrm{Cu}(\mathrm{PTQS})_{2} \mathrm{Cl}_{2}\right]$ & $1450 \mathrm{~m}, \mathrm{~b}$ & $1735 \mathrm{~m}, \mathrm{~b}$ & $530 \mathrm{~m}$ & $415 \mathrm{~m}$ & $290 \mathrm{~m}$ \\
\hline$\left[\mathrm{Cu}(\mathrm{PTQS})_{2} \mathrm{Br}_{2}\right]$ & $1450 \mathrm{~m}, \mathrm{~b}$ & $1735 \mathrm{~m}, \mathrm{~b}$ & $525 \mathrm{~m}$ & $410 \mathrm{~m}$ & $295 \mathrm{~m}$ \\
\hline$\left[\mathrm{Cu}(\mathrm{PTQS})_{2}\left(\mathrm{NO}_{3}\right)_{2}\right]$ & $1450 \mathrm{~m}, \mathrm{~b}$ & $1740 \mathrm{~m}, \mathrm{~b}$ & $530 \mathrm{~m}$ & $410 \mathrm{~m}$ & \\
\hline$\left[\mathrm{Cu}(\mathrm{PTQS})_{2}\left(\mathrm{ClO}_{4}\right)_{2}\right]$ & $1450 \mathrm{~m}, \mathrm{~b}$ & $1740 \mathrm{~m}, \mathrm{~b}$ & $530 \mathrm{~m}$ & $405 \mathrm{~m}$ & \\
\hline
\end{tabular}

${ }^{3} \mathrm{~A}_{2 \mathrm{~g}}(\mathrm{~F}),{ }^{3} \mathrm{~T}_{1 \mathrm{~g}}(\mathrm{~F}) \leftarrow{ }^{3} \mathrm{~A}_{2 \mathrm{~g}}(\mathrm{~F})$ and ${ }^{3} \mathrm{~T}_{1 \mathrm{~g}}(\mathrm{P}) \leftarrow{ }^{3} \mathrm{~A}_{2 \mathrm{~g}}(\mathrm{~F})$ indicating octahedral geometry for $\mathrm{Ni}(\mathrm{II})$ complexes. The proposed geometry of $\mathrm{Ni}(\mathrm{II})$ complexes is further supported by the $\mu_{\text {eff }}$ value in the range 3.04-3.16 BM. The $\mathrm{Cu}$ (II) complex exhibits two bands in the region 12480-12600 and 15800-16200 $\mathrm{cm}^{-1}$ assigned to the transitions ${ }^{2} \mathrm{~T}_{2 \mathrm{~g}} \leftarrow{ }^{2} \mathrm{E}_{\mathrm{g}}$ and charge transfer band which suggest octahedral geometry for the $\mathrm{Cu}(\mathrm{II})$ complex $[35,36]$. The susceptibility value of $\mathrm{Cu}(\mathrm{II})$ complex lies in the range 1.81-1.92 B.M.

\section{Conclusion}

On the basis of spectral observations, the complexes of cobalt(II), nickel(II) and copper(II) are tentatively proposed to possess octahedral geometry as shown in Fig.1.

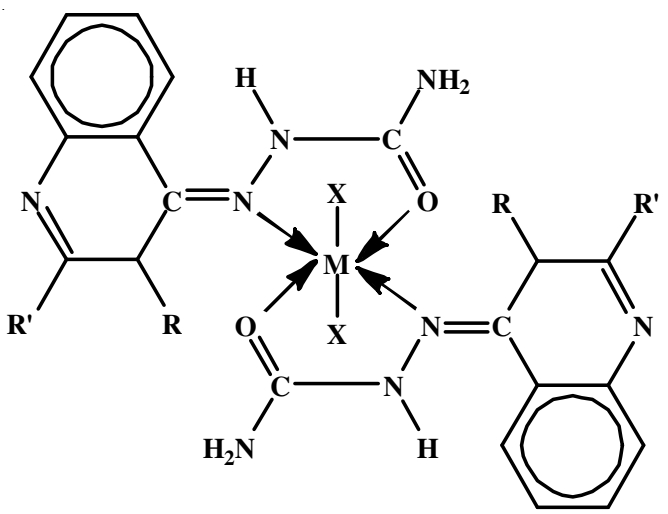

Fig. 1. $\left[\mathrm{M}(\mathrm{PTQS})_{2} \mathrm{X}_{2}\right]\left(\mathrm{M}=\mathrm{Co}^{2+}, \mathrm{Ni}^{2+}\right.$ and $\mathrm{Cu}^{2+} ; \mathrm{R}=$ propyl $\mathrm{R}^{\prime}=o$-toluidine; $\mathrm{X}=\mathrm{Cl}^{-}, \mathrm{Br}^{-}, \mathrm{I}^{-}, \mathrm{NO}_{3}^{-}$and $\mathrm{ClO}_{4}^{-}$

\section{REFERENCES}

1. R. Bastida, S. Gonzalez, T. Rodriguez, A. Sousa and D.E. Fenton, J. Chem. Soc., Dalton Trans., 3643 (1990); https://doi.org/10.1039/DT9900003643.

2. M.R. Malachowski, M. Adams, N. Elia, A.L. Rheingold and R.S. Kelly, J. Chem. Soc., Dalton Trans., 2177 (1999); https://doi.org/10.1039/A900223E.

3. L. Casella, M. Gullotti and R. Viganò, Inorg. Chim. Acta, 124, 121 (1986); https://doi.org/10.1016/S0020-1693(00)85860-0.

4. J.C. Liu, S.X. Wang, L.F. Wang, F.Y. He and X.Y. Huang, Polyhedron, 15, 3659 (1996); https://doi.org/10.1016/0277-5387(95)00523-4.
5. M.E. Hossain, M.N. Alam, M.A. Ali, M. Nazimuddin, F.E. Smith and R.C. Hynes, Polyhedron, 15, 973 (1996); https://doi.org/10.1016/0277-5387(95)00310-X

6. A.A.R. Despaigne, L.F. Vieira, I.C. Mendes, F.B. Costa, N.L. Speziali and H. Beraldo, J. Braz. Chem. Soc., 21, 1247 (2010); https://doi.org/10.1590/S0103-50532010000700012.

7. N. Nawar and N.M. Hosny, Chem. Pharm. Bull. (Tokyo), 47, 944 (1999); https://doi.org/10.1248/cpb.47.944.

8. M. Alagesan, N.S.P. Bhuvanesh and N. Dharmaraj, Dalton Trans., 42, 7210 (2013); https://doi.org/10.1039/c3dt50371b.

9. P.V. Bernhardt, G.J. Wilson, P.C. Sharpe, D.S. Kalinowski and D.R. Richardson, J. Biol. Inorg. Chem., 13, 107 (2008); https://doi.org/10.1007/s00775-007-0300-4.

10. J. Easmon, G. Purstinger, K.S. Thies, G. Heinisch and J. Hofmann, J. Med. Chem., 49, 6343 (2006); https://doi.org/10.1021/jm060232u.

11. A. Rescifina, M.G. Varrica, C. Carnovale, G. Romeo and U. Chiacchio, Eur. J. Med. Chem., 51, 163 (2012); https://doi.org/10.1016/j.ejmech.2012.02.038

12. A. Narani, R.K. Marella, P. Ramudu, K.S.R. Rao and D.R. Burri, RSC Adv., 4, 3774 (2014); https://doi.org/10.1039/C3RA44213F.

13. J.P. Collman, M. Zhong, C. Zhang and S. Costanzo, J. Org. Chem., 66, 7892 (2001); https://doi.org/10.1021/jo010615u.

14. S.Y. Ebrahimipour, I. Sheikhshoaie, M. Mohamadi, S. Suarez, R. Baggio, M. Khaleghi, M. Torkzadeh-Mahani and A. Mostafavi, Spectrochim. Acta A, 142, 410 (2015); https://doi.org/10.1016/i.saa.2015.01.088

15. M.M. Alam, G. Verma, M. Shaquiquzzaman, A. Marella, M. Akhtar and M.R. Ali, J. Pharm. Bioallied Sci., 6, 69 (2014); https://doi.org/10.4103/0975-7406.129170.

16. D. Dey, S. Pal, P.P. Bag, S. Saha and S. Chandraleka, J. Indian Chem. Soc., 92, 191 (2017).

17. N. Gayathri and M.S. Suresh, Asian J. Chem., 29, 541 (2017); https://doi.org/10.14233/ajchem.2017.20227.

18. J. Devi and M. Yadav, Asian J. Chem., 29, 551 (2017); https://doi.org/10.14233/ajchem.2017.20236.

19. A. Ahlawat, S. Asija and N. Singh, Asian J. Chem., 29, 669 (2017); https://doi.org/10.14233/ajchem.2017.20303.

20. N.T. Dhokale, B.K. Karale and A.V. Nagawade, Asian J. Chem., 29, 843 (2017); https://doi.org/10.14233/ajchem.2017.20329.

21. B.K. Rai and A. Kumar, Asian J. Chem., 25, 1169 (2013); https://doi.org/10.14233/ajchem.2013.13199A.

22. B.K. Rai, J. Indian Chem. Soc., 90, 105 (2013).

23. R. Rai, R.R. Kumar, M. Kumar and B.K. Rai, Orient. J. Chem., 30, 303 (2014); https://doi.org/10.13005/ojc/300139. 
24. R. Kumari and B.K. Rai, Asian J. Chem., 26(Suppl.), S280 (2014); https://doi.org/10.14233/ajchem.2014.19065.

25. R. Rai, A. Baluni, P. Kumari and B.K. Rai, Asian J. Chem., 27, 2237 (2015); https://doi.org/10.14233/ajchem.2015.18640.

26. A.I. Vogel, A Textbook of Quantitative Chemical Analysis, Revised by J. Bessett, R.C. Denny, J.H. Jeffery and J. Mendham, ELBS, London, edn 5 (1997).

27. S. Silverstein and X.W. Francis, Spectrometric Identification of Organic Compounds, John Wiley \& Sons, end 6 (2008).

28. W. Kemp, Organic Spectroscopy, Palgrove, New York, edn 3 (2008).

29. K. B. Gudasi, S. A. Patil, J. Serb. Chem. Soc., 17, 520 (2006).

30. P.P. Singh and V.P. Shukla, Inorg. Chim. Acta, 7, 493 (1973); https://doi.org/10.1016/S0020-1693(00)94870-9.
31. J.R. Ferraro, Low Frequency Vibration of Inorganic and Coordination Compound, Plenum Press, New York (1971).

32. D.M. Boghaei and N. Lashanizadegan, Synth React. Inorg. Metal-Org. Chem., 30, 1393 (2000); https://doi.org/10.1080/00945710009351841.

33. C.C. Addition, N. Logan, S.C. Wallwork and D.C. Garner, Quart. Rev., 25, 289 (1971).

34. H.L. Harikumaran Nair and C.P. Prathakaran, Indian J. Chem., 39A, 989 (2000).

35. B.N. Figgis, Introduction to Ligand Field, Wiley Eastern Ltd., New Delhi, p. 279 (1976)

36. R.N. Carlin and A.J. Van bryneveledt, Magnetic Properties of Transition Metal Complexes, Springer Verlag, New York (1997). 